\title{
Genetic effects of rs3740199 polymorphism in ADAM12 gene on knee osteoarthritis: a meta-analysis
}

\author{
Zheng $\mathrm{Hao}^{1 \dagger}$, Xin $\mathrm{Li}^{2 \dagger}$, Jin Dai ${ }^{3,4}$, Baocheng Zhao ${ }^{1 *}$ and Qing Jiang ${ }^{3,4^{*}}$
}

\begin{abstract}
Background: Knee osteoarthritis $(\mathrm{OA})$ is a complex arthritic condition in which genetic factors play an important role. ADAM12 gene is one of the recognized candidate genes although the results are conflicting. To derive a more precise estimation of the association between rs3740199 polymorphism in ADAM12 gene and risk of knee OA, we performed a meta-analysis based on six related studies, including a total of 2185 cases and 3716 controls.

Methods: A comprehensive search was performed to identify related studies up to April 14, 2017. We used odds ratios (ORs) with 95\% confidence intervals (Cls) to assess the strength of the association. Different genetic models were used to assess the pooled and stratified data.

Results: Overall, no significant association was found in all genetic models (C vs. G, OR $=0.983,95 \% \mathrm{Cl}=0.910-1$. 061; $C C$ vs. GG, $O R=1.033,95 \% \mathrm{Cl}=0.851-1.255 ; \mathrm{CG}$ vs. $\mathrm{GG}, \mathrm{OR}=1.030,95 \% \mathrm{Cl}=0.877-1.209 ; \mathrm{CC} / \mathrm{CG}$ vs. $\mathrm{GG}, \mathrm{OR}=1$. $031,95 \% \mathrm{Cl}=0.886-1.201$; $\mathrm{CC}$ vs. $\mathrm{CG} / \mathrm{GG}, \mathrm{OR}=1.017,95 \% \mathrm{Cl}=0.868-1.190)$. When stratified by ethnicity, no significant association was found.
\end{abstract}

Conclusions: This meta-analysis suggested that the rs3740199 polymorphism does not contribute to the development of knee OA. Additional well-designed large studies are required to confirm these findings in different populations.

Keywords: Osteoarthritis, ADAM12, rs3740199, Polymorphism, Meta-analysis

\section{Background}

Osteoarthritis (OA) is a complex arthritic condition characterized by progressive cartilage loss, synovitis, osteophyte formation, and subchondral sclerosis. It is a cause of important handicap among the elderly $[1,2]$. It has been reported that there were nearly 85 million OA patients in the world in 2009, and it might increase to 122 million in 2017. Hence, it is an enormous burden on the national economy and healthcare system $[3,4]$. OA is a multifactorial disease resulting from the combined influence of environmental factors and genes [5]. Age,

\footnotetext{
*Correspondence: drzhaobaocheng@outlook.com; jiangqing112@hotmail.com ${ }^{\dagger}$ Equal contributors

${ }^{1}$ Center of Diagnosis and Treatment for Developmental Dysplasia of the Hip, Nanjing Zhongyangmen Community Health Service Center, Kang'ai Hospital, Nanjing 210037, Jiangsu, People's Republic of China

${ }^{3}$ Department of Sports Medicine and Adult Reconstructive Surgery, Drum Tower Hospital, School of Medicine, Nanjing University, 321 Zhongshan Road, Nanjing 210008, Jiangsu, People's Republic of China

Full list of author information is available at the end of the article
}

joint injury, and obesity are the major risk factors $[6,7]$. Several studies have identified some genetic factors such as ASPN [8], FRZB [9], and GDF5 [10]. These three genes are involved in controlling growth and differentiation pathways [11]. Many other polymorphisms have shown association to OA although the results are inconsistent. Further research is needed to replicate these findings and identify some new genetic factors [12].

A disintegrin and metalloprotease (ADAM), a member of the $\mathrm{Zn}$-dependent metzincin superfamily, is associated with many complex diseases such as heart disease, rheumatoid arthritis, Alzheimer's disease, and cancer [13, 14]. ADAM12 may play an important role in chondrocyte proliferation, maturation, bone formation, and osteoclast differentiation [15-18]. ADAM12 is up-regulated in multinucleated giant cells surrounding loose hip implants and OA cartilage $[19,20]$. Recently, promising but contradictory data have been published for the association of ADAM12 with OA [21-25]. Poonpet et al. [24] and Kerna et al. 
[25] found that rs3740199 in ADAM12 was associated with knee OA risk although the results were conflicting rather than conclusion [12, 23, 26, 27].

In the present study, a meta-analysis was performed to determine the overall association between ADAM12 rs3740199 polymorphism and knee OA susceptibility and whether the association varies by ethnicity.

\section{Methods}

\section{Literature search strategy}

To identify all relevant reports on rs3740199 polymorphism and knee OA risk, we performed a systematic search for all English language papers from PubMed (the last search update was April 14, 2017), using the key words "rs3740199" or "ADAM12," "polymorphism" or "polymorphisms" or "SNP," "osteoarthritis" or "OA". Additional eligible studies were identified by a manual search of the references of retrieved studies and review articles.

According to the following criteria, six studies were included in this meta-analysis: (1) was a cohort or casecontrol study; (2) was a study of the ADAM12 rs3740199 polymorphism and knee OA risk; and (3) contained available genotype or allele frequency of rs3740199.

\section{Data extraction}

Two of the investigators extracted all data independently according to the criteria described above. We developed a data extraction sheet including year of publication, the first author's name, OA type, country of origin, ethnicity, assessment of OA, genotyping method, source of control groups, genotype, and allele frequency. For studies contain the results from different knee OA types, each type was treated independently. Any controversies of the data were discussed within our research team and the authors reached a consensus on all items.

\section{Statistical methods}

Allele frequencies of the ADAM12 rs3740199 polymorphism from the six eligible studies were calculated by the allele counting method respectively. Hardy-Weinberg equilibrium (HWE) was used to evaluate the deviation of data associated with the ADAM12 rs3740199 SNP in the control groups using $X^{2}$ test. The strength of association between the ADAM12 rs3740199 polymorphism and knee OA susceptibility was evaluated by pooled odds ratios (ORs) and their 95\% confidence intervals (CIs). The significance of the ORs and 95\% CIs was determined by $Z$ test. The pooled ORs and 95\% CIs were performed for additive model ( $\mathrm{C}$ vs. $\mathrm{G}$ ), co-dominant model (CC vs. GG; CG vs. GG), dominant model (CC/CG vs. GG), and recessive model (CC vs. CG/GG). Stratified analysis was also performed by ethnicity.

We assessed the between-study heterogeneity using chi-square-based $Q$ test. If the $P$ value was less than
0.10 , the heterogeneity was considered significant. We also used the $I^{2}$ statistic $\left(I^{2}=100 \% \times(Q-d f) / \mathrm{Q}\right)$ to quantify heterogeneity. $I^{2}$ greater than $50 \%$ indicated the presence of heterogeneity among studies. The fixedeffects model based on the Mantel-Haenszel method and the random-effects model based on the Dersimonian and Laird method were used to pool the data [28]. The random-effects model was more appropriate in the presence of heterogeneity; otherwise, the two methods provide similar results.

In meta-analysis, publication bias is also a concern. To test for publication bias, both Egger's and Begg's test are commonly used [29]. In this study, publication bias was evaluated by funnel plot and the linear regression asymmetry test.

All analyses were carried out using Stata software version 8.2 (Stata Corporation, College Station, TX, USA). All tests were two-sided.

\section{Results}

\section{Characteristics of the included studies}

Eleven relevant studies identified and screened. Four studies were added through manual search of the reference lists of retrieved studies. Nine of the 15 studies were excluded: three not polymorphism, one not for OA research, and five no useable data reported. A total of six reports were identified [12, 23-27]. Among these, Rodriguez-Lopez et al. reported six sample collections [12] while Kerna et al. included subjects with tibiofemoral knee OA (TFOA) and patellofemoral knee OA (PFOA) [25], they were considered as independent studies. Finally, nine studies with 2185 cases and 3716 controls were included in the present meta-analysis. The detailed study flow chart was illustrated in Fig. 1. Characteristics of the nine studies were listed in Tables 1 and 2. Of eligible studies, four and five studies were conducted in Asian and European populations respectively.

\section{Quantitative synthesis}

The details of meta-analysis for ADAM12 rs3740199 polymorphism with knee OA risk are shown in Table 3.

\section{Overall population}

Nine separate studies with a total sample size of 2185 cases and 3716 controls had available data for analyzing the association of ADAM12 rs3740199 polymorphism and knee OA risk. No significant association was found in all genetic models (C vs. G, OR $=0.983$, 95\% $\mathrm{CI}=0.910-1.061 ; \mathrm{CC}$ vs. $\mathrm{GG}, \mathrm{OR}=1.033,95 \% \mathrm{CI}=$ $0.851-1.255$; $C G$ vs. $G G, O R=1.030,95 \% \mathrm{CI}=0.877-$ 1.209 ; $\mathrm{CC} / \mathrm{CG}$ vs. $\mathrm{GG}, \mathrm{OR}=1.031,95 \% \mathrm{CI}=0.886-$ 1.201; $\mathrm{CC}$ vs. $\mathrm{qECG} / \mathrm{GG}, \mathrm{OR}=1.017,95 \% \mathrm{CI}=0.868$ 1.190) (Table 3). 


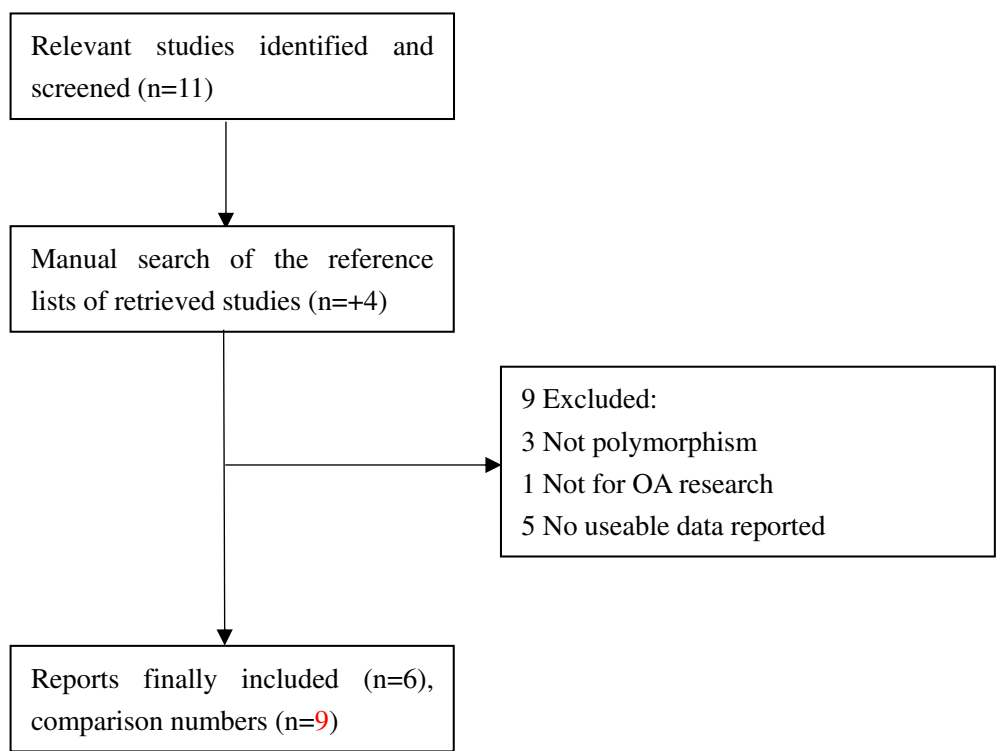

Fig. 1 Studies identified with criteria for inclusion and exclusion

\section{Subgroup analysis by ethnicity}

No significant association was found in all genetic models among Asian population ( $\mathrm{C}$ vs. $\mathrm{G}, \mathrm{OR}=1.020$, 95\% $\mathrm{CI}=0.924-1.127$; $\mathrm{CC}$ vs. $\mathrm{GG}, \mathrm{OR}=1.040,95 \% \mathrm{CI}=$ $0.850-1.272$; $\mathrm{CG}$ vs. $\mathrm{GG}, \mathrm{OR}=1.035,95 \% \mathrm{CI}=0.878-$ 1.221 ; $\mathrm{CC} / \mathrm{CG}$ vs. $\mathrm{GG}, \mathrm{OR}=1.036,95 \% \mathrm{CI}=0.887-$ 1.211 ; $\mathrm{CC}$ vs. CG/GG, OR $=1.017,95 \% \mathrm{CI}=0.858-$ 1.207). No significant association was found in European population either.

\section{Heterogeneity and publication bias}

The between-study heterogeneity of the ADAM12 rs3740199 polymorphism was not found in all subjects, and thus, the meta-analysis of the ADAM12 rs3740199 polymorphism was performed using a fixed-effects model for all subjects except for the recessive model in European population, which was analyzed using a random-effects model $\left(I^{2}=73.4 \%\right)$ (Table 3$)$.
In this study, publication bias was evaluated by funnel plot and the linear regression asymmetry test. As shown in Fig. 2, the shape of the funnel did not reveal obvious asymmetry. Then, Egger's test was then performed to estimate the funnel plot symmetry. The results still did not show any evidence of publication bias $(t=-0.40, P=$ 0.699 for $\mathrm{C}$ vs. G).

\section{Discussion}

OA is well recognized as a multifactorial disease. In addition to age, sex, trauma, and body weight, genetic factors are also strong determinants of this disease [30]. More than $50 \%$ of the OA cases can be attributed to genetic factors, demonstrated by twins and family studies [31]. It is suggested that OA of the hand, spine, hip, and knee are all heritable [32]. Recently, genetic studies have found many genes are contributing to OA, although with relatively modest effect [33]. These observations have

Table 1 Characteristics of literatures included in this meta-analysis

\begin{tabular}{|c|c|c|c|c|c|c|c|c|c|}
\hline Year & First author & OA type & Country & Ethnicity & Assessment of OA & Genetyping & Source of controls & Cases & Controls \\
\hline \multirow[t]{3}{*}{2009} & J. Rodriguez-Lopez & TKR & Spain & European & KL score & Multiplex single-base extension & PB & 262 & 294 \\
\hline & & TKR & UK & European & K/L score & Multiplex single-base extension & PB & 360 & 698 \\
\hline & & TKR & Greece & European & KL score & Multiplex single-base extension & PB & 159 & 193 \\
\hline \multirow[t]{2}{*}{2009} & I. Kerna & TFOA & Estonian & European & OA score & PCR-RFLP & PB & 66 & 123 \\
\hline & & PFOA & Estonian & European & OA score & PCR-RFLP & PB & 97 & 92 \\
\hline 2012 & Min-Ho Shin & Knee OA & Korea & Asian & KLL score & TaqMan & PB & 725 & 1737 \\
\hline 2014 & Suliang Lou & Knee OA & China & Asian & KLL score & TaqMan & PB & 152 & 179 \\
\hline 2015 & LinWang & Knee OA & China & Asian & KLL score & iMLDR & PB & 164 & 200 \\
\hline 2016 & Thitiya Poonpet & Knee OA & Thai & Asian & KLL score & HRM analysis & PB & 200 & 200 \\
\hline
\end{tabular}

$K / L$ score Kellgren-Lawrence score, $P B$ population based, TKR total knee replacement, TFOA tibiofemoral knee OA, $P C R$-RFLP polymerase chain reaction-restriction fragment length polymorphism, $P F O A$ patellofemoral knee OA, iMLDR improved multiplex ligase detection reaction, $H R M$ high resolution melting 
Table 2 Distributions of ADAM2 rs3740199 genotypes and alleles among cases and controls

\begin{tabular}{|c|c|c|c|c|c|c|c|c|c|c|c|c|}
\hline \multirow[t]{2}{*}{ Year } & \multirow[t]{2}{*}{ First author } & \multicolumn{3}{|l|}{ Case } & \multicolumn{3}{|c|}{ Control } & \multicolumn{2}{|c|}{ Case } & \multicolumn{2}{|c|}{ Control } & \multirow{2}{*}{$\begin{array}{l}\text { HWE in } \\
\text { control }\end{array}$} \\
\hline & & $\mathrm{CC}$ & GC & GG & $\mathrm{CC}$ & GC & GG & $\mathrm{C}$ & G & $\mathrm{C}$ & G & \\
\hline 2009 & J. Rodriguez-Lopez & NA & NA & NA & NA & NA & NA & 290 & 234 & 327 & 261 & NA \\
\hline 2009 & J. Rodriguez-Lopez & NA & NA & NA & NA & NA & NA & 370 & 350 & 744 & 652 & NA \\
\hline 2009 & J. Rodriguez-Lopez ${ }^{a}$ & NA & NA & NA & NA & NA & NA & 180 & 138 & 239 & 147 & NA \\
\hline 2009 & I. Kerna ${ }^{a}$ & 28 & 32 & 6 & 65 & 46 & 12 & 88 & 44 & 176 & 70 & 0.366 \\
\hline 2009 & I. Kerna ${ }^{a}$ & 53 & 34 & 10 & 41 & 43 & 8 & 140 & 54 & 125 & 59 & 0.485 \\
\hline 2012 & Min-Ho Shin & 147 & 364 & 214 & 350 & 863 & 524 & 658 & 792 & 1563 & 1911 & 0.876 \\
\hline 2014 & Suliang Lou & 32 & 78 & 42 & 42 & 93 & 44 & 142 & 162 & 177 & 181 & 0.6 \\
\hline 2015 & LinWang & 36 & 84 & 44 & 47 & 102 & 51 & 156 & 172 & 196 & 204 & 0.773 \\
\hline 2016 & Thitiya Poonpet & 56 & 102 & 42 & 46 & 100 & 54 & 214 & 186 & 192 & 208 & 0.982 \\
\hline
\end{tabular}

HWE Hardy-Weinberg equilibrium, NA data not available

${ }^{a} A n$ independent study in one article

encouraged us to search for more responsible genes. Many genes have been studied and the ADAM12 gene is one of the possible candidate genes for OA [12, 23-27]. Some investigations suggested a regulatory role of $A D A M 12$ in chondrocyte proliferation, maturation, bone formation, and osteoclast differentiation [15-18]. Association studies have been arranged to investigate the role of a nonsynonymous polymorphism (rs3740199) in the second exon of ADAM12 in knee OA risk that has been reported to date [12, 21, $25,26,34]$. However, these findings have been inconsistent and contradictory.
Meta-analysis is a suitable method to combine the results of individual studies, overcome the disadvantages of a single study, and increase the statistical power. The present study was to investigate and update the results associating the ADAM12 rs3740199 polymorphism with knee OA risk in different ethnic populations. To our knowledge, the present study is the first meta-analysis which estimated the association between ADAM12 rs3740199 and knee OA susceptibility. No significant association of ADAM12 rs3740199 polymorphisms with knee OA risk was demonstrated in our study. We also failed to find the association between knee OA and the

Table 3 Meta-analysis for the ADAM2 rs3740199 polymorphism and knee OA risk

\begin{tabular}{|c|c|c|c|c|c|}
\hline \multirow[t]{2}{*}{ Population } & \multirow[t]{2}{*}{ Comparison $\left(N^{a}\right)$} & \multicolumn{2}{|l|}{ Test of association } & \multicolumn{2}{|c|}{ Test of heterogeneity } \\
\hline & & OR $(95 \% \mathrm{Cl})$ & $p^{b}$ & $\overline{p^{c}}$ & $P^{2}(\%)$ \\
\hline \multirow[t]{5}{*}{ Overall } & C vs. G (12) & $0.983(0.910-1.061)$ & 0.657 & 0.490 & 0.0 \\
\hline & CC vs. GG (6) & $1.033(0.851-1.255)$ & 0.740 & 0.681 & 0.0 \\
\hline & CG vs. GG (6) & $1.030(0.877-1.209)$ & 0.721 & 0.770 & 0.0 \\
\hline & CC/CG vs. GG (6) & $1.031(0.886-1.201)$ & 0.690 & 0.768 & 0.0 \\
\hline & CC vs. CG/GG (6) & 1.017 (0.868-1.190) & 0.837 & 0.364 & 8.1 \\
\hline \multicolumn{6}{|l|}{ Ethnicity } \\
\hline \multirow[t]{5}{*}{ Asian } & C vs. G (4) & $1.020(0.924-1.127)$ & 0.689 & 0.398 & 0.0 \\
\hline & CC vs. GG (4) & $1.040(0.850-1.272)$ & 0.703 & 0.390 & 0.4 \\
\hline & CG vs. GG (4) & $1.035(0.878-1.221)$ & 0.681 & 0.709 & 0.0 \\
\hline & CC/CG vs. GG (4) & $1.036(0.887-1.211)$ & 0.656 & 0.502 & 0.0 \\
\hline & CC vs. CG/GG (4) & $1.017(0.858-1.207)$ & 0.843 & 0.642 & 0.0 \\
\hline \multirow[t]{5}{*}{ European } & C vs. G (8) & $0.930(0.825-1.049)$ & 0.239 & 0.538 & 0.0 \\
\hline & CC vs. GG (2) & $0.950(0.453-1.992)$ & 0.891 & 0.809 & 0.0 \\
\hline & CG vs. GG (2) & $0.927(0.446-1.930)$ & 0.840 & 0.301 & 6.6 \\
\hline & CC/CG vs. GG (2) & $0.940(0.465-1.901)$ & 0.864 & 0.713 & 0.0 \\
\hline & CC vs. CG/GG (2) & $0.998(0.445-2.237)$ & 0.996 & 0.052 & 73.4 \\
\hline
\end{tabular}

$O R$ odds ratio, $\mathrm{Cl}$ confidence interval

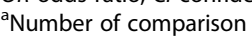

${ }^{\mathrm{b}} P$ values for within group differences were determined by $Z$ test

${ }^{c} P$ value of $Q$ test for heterogeneity test 
Begg's funnel plot with pseudo 95\% confidence limits

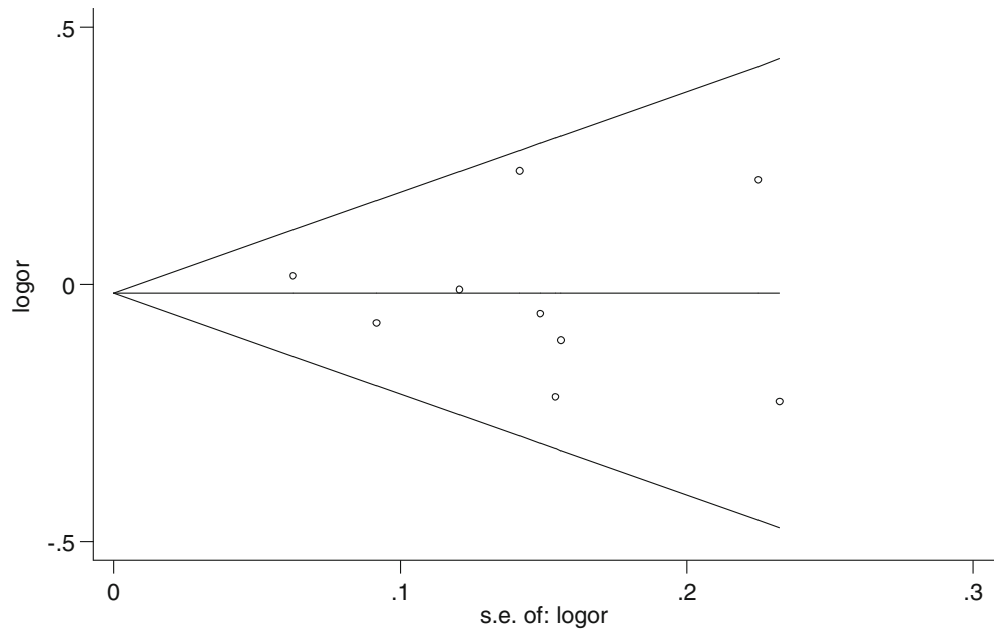

Fig. 2 Begg's funnel plot for publication bias test (C versus G). Each point represented a separate study for the indicated association. logor natural logarithm of OR. Horizontal line mean effect size

ADAM12 polymorphism in Asian and European population. No heterogeneity was found in overall population and Asian population, while high heterogeneity was seen in the recessive model in European population.

Association studies with complex outcomes for detecting genetic variants must be considered with caution because the results may be influenced by many factors. Our present study showed a lack of association between the ADAM12 rs3740199 polymorphism and knee OA risk, which is not consistent with the association or functional studies of the ADAM12 polymorphism. However, epidemiologic results are always different from the functional studies because $\mathrm{OA}$ is a multifactorial disease influenced by different genetic backgrounds, multiple genes, and environmental factors. Our negative results of the ADAM12 polymorphism may also be due to type II error. In recent years, some genome-wide association studies (GWAS) have already reported GDF5, BTNL2, DUS4L, COG5, SENP6, and FILIP1 as OA candidate genes [35, 36]. However, the ADAM12 polymorphism has not been confirmed in these GWAS [35, 36].

Our results are consistent with some studies that also failed to detect association between rs3740199 and OA susceptibility in either male or female patients [12, 23, $26,27]$. On the other hand, Poonpet et al. reported the rs3740199 polymorphism was associated with knee OA risk, while the effect was only found in Thai male patients [24]. The C allele of rs3740199 was found to be associated with OA in female patients in the UK [21]. Kerna et al. found significant association between ADAM12 rs3740199 and PFOA in male patients [25]. The reason for the contradictory results remain unclear, but the differences in study populations including age, gender, sample size, and disease severity may play an important role. In the meanwhile, the environmental factors in each sample such as lifestyles, diets, and selected physical activity may also affect the association between rs3740199 polymorphism and OA risk. Lastly, each population has their own gene pool, so it is not surprising that there are differences in the distributions of $A D A M 12$ rs3740199 genotypes and alleles from subjects with different ethnicities.

Some limitations of this meta-analysis should also be noted. First, the potential confounding factors (such as age, gender) were not adjusted in the present study. Second, the gene-environment interactions and the effect of gene-gene should also be considered because they might influence the biological effects of the polymorphisms of the $A D A M 12$ gene. Third, because it was difficult to get all full papers published in different languages, we only included six studies published in English language. Fourth, the included subjects were not adequate to confirm a robust conclusion and the association should be resolved by larger studies.

\section{Conclusions}

In conclusion, this meta-analysis did not reveal any association between the ADAM12 rs3740199 polymorphism and knee OA risk. Additional larger studies are needed to confirm our findings in the future.

\section{Abbreviations}

ADAM: A disintegrin and metalloprotease; Cls: Confidence intervals; GWAS: Genome-wide association studies; HRM: High resolution melting; HWE: Hardy-Weinberg equilibrium; iMLDR: Improved multiplex ligase detection reaction; K/L score: Kellgren-Lawrence score; OA: Osteoarthritis; ORs: Odds ratios; PB: Population based; PCR-RFLP: Polymerase chain reactionrestriction fragment length polymorphism; PFOA: Patellofemoral knee OA; TFOA: Tibiofemoral knee OA; TKR: Total knee replacement 


\section{Acknowledgements}

This work was supported by the Projects of International Cooperation and Exchanges NSFC (81420108021), National Key Technology Support Program (2015BAI08B02), Excellent Young Scholars NSFC (81622033), Jiangsu Provincial Key Medical Center Foundation, Jiangsu Provincial Medical Talent Foundation, and Jiangsu Provincial Medical Outstanding Talent Foundation. We thank the patients and their families who donated their blood samples for this study.

\section{Funding}

Not applicable.

\section{Availability of data and materials}

As this paper is a meta-analysis, there are no patient data sets. The search strategy was for the study selection, which supports the conclusion of the meta-analysis.

\section{Authors' contributions}

$\mathrm{BZ}, \mathrm{QJ}$ contributed to the critical revision of the article. $\mathrm{ZH}, \mathrm{XL}$ conceived of the design of the study. ZH, XL and JD performed and collected the data and contributed to the design of the study. ZH, XL and JD prepared and revised the manuscript. All authors read and approved the final content of the manuscript.

\section{Competing interests}

The authors declare that they have no competing interests.

\section{Consent for publication}

Not applicable.

Ethics approval and consent to participate

Not applicable.

\section{Publisher's Note}

Springer Nature remains neutral with regard to jurisdictional claims in published maps and institutional affiliations.

\section{Author details}

'Center of Diagnosis and Treatment for Developmental Dysplasia of the Hip, Nanjing Zhongyangmen Community Health Service Center, Kang'ai Hospital, Nanjing 210037, Jiangsu, People's Republic of China. ${ }^{2}$ Department of HIV/ AIDS/STI Prevention and Control, Nanjing Municipal Center for Diseases Control and Prevention, Nanjing 210009, Jiangsu, People's Republic of China. ${ }^{3}$ Department of Sports Medicine and Adult Reconstructive Surgery, Drum Tower Hospital, School of Medicine, Nanjing University, 321 Zhongshan Road, Nanjing 210008, Jiangsu, People's Republic of China. ${ }^{4}$ Laboratory for Bone and Joint Disease, Model Animal Research Center (MARC), Nanjing University, Nanjing 210093, Jiangsu, China.

\section{Received: 15 April 2017 Accepted: 3 June 2017}

Published online: 20 June 2017

\section{References}

1. Zhuo Q, Yang W, Chen J, Wang Y. Metabolic syndrome meets osteoarthritis. Nat Rev Rheumatol. 2012;8:729-37.

2. Felson DT. Developments in the clinical understanding of osteoarthritis. Arthritis Res Ther. 2009;11:203.

3. Litwic A, Edwards MH, Dennison EM, Cooper C. Epidemiology and burden of osteoarthritis. Br Med Bull. 2013;105:185-99.

4. Cross M, Smith E, Hoy D, Nolte S, Ackerman I, Fransen M, et al. The global burden of hip and knee osteoarthritis: estimates from the global burden of disease 2010 study. Ann Rheum Dis. 2014;73:1323-30.

5. Johnson VL, Hunter DJ. The epidemiology of osteoarthritis. Best Pract Res Clin Rheumatol. 2014;28:5-15.

6. Guilak F. Biomechanical factors in osteoarthritis. Best Pract Res Clin Rheumatol. 2011;25:815-23.

7. van Tunen JA, Dell'Isola A, Juhl C, Dekker J, Steultjens M, Lund H. Biomechanical factors associated with the development of tibiofemoral knee osteoarthritis: protocol for a systematic review and meta-analysis. BMJ Open. 2016;6:e11066.
8. Kizawa H, Kou I, lida A, Sudo A, Miyamoto Y, Fukuda A, et al. An aspartic acid repeat polymorphism in asporin inhibits chondrogenesis and increases susceptibility to osteoarthritis. Nat Genet. 2005;37:138-44.

9. Loughlin J, Dowling B, Chapman K, Marcelline L, Mustafa Z, Southam L, et al. Functional variants within the secreted frizzled-related protein 3 gene are associated with hip osteoarthritis in females. Proc Natl Acad Sci U S A. 2004; 101:9757-62.

10. Miyamoto Y, Mabuchi A, Shi D, Kubo T, Takatori Y, Saito S, et al. A functional polymorphism in the 5' UTR of GDF5 is associated with susceptibility to osteoarthritis. Nat Genet. 2007;39:529-33.

11. Nakajima M, Kizawa H, Saitoh M, Kou I, Miyazono K, Ikegawa S. Mechanisms for asporin function and regulation in articular cartilage. J Biol Chem. 2007; 282:32185-92.

12. Rodriguez-Lopez J, Pombo-Suarez M, Loughlin J, Tsezou A, Blanco FJ, Meulenbelt I, et al. Association of a nsSNP in ADAMTS14 to some osteoarthritis phenotypes. Osteoarthritis Cartilage. 2009;17:321-7.

13. Kveiborg M, Albrechtsen R, Couchman JR, Wewer UM. Cellular roles of ADAM12 in health and disease. Int J Biochem Cell Biol. 2008;40:1685-702.

14. Mochizuki S, Okada Y. ADAMs in cancer cell proliferation and progression. Cancer Sci. 2007;98:621-8.

15. Verrier S, Hogan A, McKie N, Horton M. ADAM gene expression and regulation during human osteoclast formation. Bone. 2004;35:34-46.

16. Abe E, Mocharla H, Yamate T, Taguchi Y, Manolagas SC. Meltrin-alpha, a fusion protein involved in multinucleated giant cell and osteoclast formation. Calcif Tissue Int. 1999;64:508-15.

17. Kveiborg M, Albrechtsen R, Rudkjaer L, Wen G, Damgaard-Pedersen $K$, Wewer UM. ADAM12-S stimulates bone growth in transgenic mice by modulating chondrocyte proliferation and maturation. J Bone Miner Res. 2006;21:1288-96.

18. Okada A, Mochizuki S, Yatabe T, Kimura T, Shiomi T, Fujita Y, et al. ADAM-12 (meltrin alpha) is involved in chondrocyte proliferation via cleavage of insulin-like growth factor binding protein 5 in osteoarthritic cartilage. Arthritis Rheum. 2008;58:778-89.

19. Sato T, Konomi K, Yamasaki S, Aratani S, Tsuchimochi K, Yokouchi M, et al. Comparative analysis of gene expression profiles in intact and damaged regions of human osteoarthritic cartilage. Arthritis Rheum. 2006;54:808-17.

20. Ma G, Ainola M, Liljestrom M, Santavirta S, Poduval P, Zhao D, et al. Increased expression and processing of ADAM 12 (meltrin-alpha) in osteolysis associated with aseptic loosening of total hip replacement implants. J Rheumatol. 2005;32:1943-50.

21. Valdes AM, Hart DJ, Jones KA, Surdulescu G, Swarbrick P, Doyle DV, et al. Association study of candidate genes for the prevalence and progression of knee osteoarthritis. Arthritis Rheum. 2004;50:2497-507.

22. Limer KL, Tosh K, Bujac SR, McConnell R, Doherty S, Nyberg F, et al. Attempt to replicate published genetic associations in a large, well-defined osteoarthritis case-control population (the GOAL study). Osteoarthritis Cartilage. 2009;17:782-9.

23. Lou S, Zhao Z, Qian J, Zhao K, Wang R. Association of single nucleotide polymorphisms in ADAM12 gene with susceptibility to knee osteoarthritis: a case-control study in a Chinese Han population. Int J Clin Exp Pathol. 2014; 7:5154-9.

24. Poonpet T, Tammachote R, Tammachote N, Kanitnate S, Honsawek S Association between ADAM12 polymorphism and knee osteoarthritis in Thai population. Knee. 2016;23:357-61.

25. Kerna I, Kisand K, Tamm AE, Lintrop M, Veske K, Tamm AO. Missense single nucleotide polymorphism of the ADAM12 gene is associated with radiographic knee osteoarthritis in middle-aged Estonian cohort. Osteoarthritis Cartilage. 2009;17:1093-8.

26. Shin MH, Lee SJ, Kee SJ, Song SK, Kweon SS, Park DJ, et al. Genetic association analysis of GDF5 and ADAM12 for knee osteoarthritis. Joint Bone Spine. 2012;79:488-91.

27. Wang L, Guo L, Tian F, Hao R, Yang T. Analysis of single nucleotide polymorphisms within ADAM12 and risk of knee osteoarthritis in a Chinese Han population. Biomed Res Int. 2015;2015:518643.

28. MANTEL N, HAENSZEL W. Statistical aspects of the analysis of data from retrospective studies of disease. J Natl Cancer Inst. 1959;22:719-48.

29. Egger M, Davey SG, Schneider M, Minder C. Bias in meta-analysis detected by a simple, graphical test. BMJ. 1997;315:629-34.

30. Lee YH, Rho YH, Choi SJ, Ji JD, Song GG. Osteoarthritis susceptibility loci defined by genome scan meta-analysis. Rheumatol Int. 2006;26:959-63. 
31. MacGregor AJ, Spector TD. Twins and the genetic architecture of osteoarthritis. Rheumatology (Oxford). 1999;38:583-8.

32. Bijkerk C, Houwing-Duistermaat JJ, Valkenburg HA, Meulenbelt I, Hofman A, Breedveld FC, et al. Heritabilities of radiologic osteoarthritis in peripheral joints and of disc degeneration of the spine. Arthritis Rheum. 1999:42:1729-35.

33. Valdes AM, Doherty M, Spector TD. The additive effect of individual genes in predicting risk of knee osteoarthritis. Ann Rheum Dis. 2008;67:124-7.

34. Valdes AM, Van Oene M, Hart DJ, Surdulescu GL, Loughlin J, Doherty M, et al. Reproducible genetic associations between candidate genes and clinical knee osteoarthritis in men and women. Arthritis Rheum. 2006;54:533-9.

35. Zeggini E, Panoutsopoulou K, Southam L, Rayner NW, Day-Williams AG, Lopes MC, et al. Identification of new susceptibility loci for osteoarthritis (arcOGEN): a genome-wide association study. Lancet. 2012;380:815-23.

36. Evangelou E, Valdes AM, Kerkhof HJ, Styrkarsdottir U, Zhu Y, Meulenbelt I, et al. Meta-analysis of genome-wide association studies confirms a susceptibility locus for knee osteoarthritis on chromosome 7q22. Ann Rheum Dis. 2011;70:349-55.

\section{Submit your next manuscript to BioMed Central} and we will help you at every step:

- We accept pre-submission inquiries

- Our selector tool helps you to find the most relevant journal

- We provide round the clock customer support

- Convenient online submission

- Thorough peer review

- Inclusion in PubMed and all major indexing services

- Maximum visibility for your research

Submit your manuscript at www.biomedcentral.com/submit 